\title{
Spinal Dural Arteriovenous Fistula
}

\section{Stéphanie Lenck ${ }^{1,3 *}$, Anne-Laure Bernat ${ }^{2}$, Damien Bresson ${ }^{2}$, Marc-Antoine Labeyrie ${ }^{1,3}$, Jean-Pierre Saint-Maurice ${ }^{1,3}$, Sébastien Froelich ${ }^{2}$ and} Emmanuel Houdart ${ }^{1,3}$

${ }^{1}$ Department of Neuroradiology, Hôpital Lariboisière, 2 rue Ambroise Paré, 75010 Paris, France

${ }^{2}$ Department of Neurosurgery, Hôpital Lariboisière, 2 rue Ambroise Paré, 75010 Paris, France

${ }^{3}$ EA 7334 REMES, Université Paris-Diderot, Paris, France

\begin{abstract}
Spinal dural arteriovenous fistulas are a rare and underdiagnosed pathology. Rapid diagnosis and treatment are, however, major determining factors for the outcome of the disease. The spontaneous course of this pathology can result in a substantial degree of morbidity that may be irreversible. Proper interpretation of the MRI imaging, and particularly the medullar angiography, allow for an appropriate level of therapeutic intervention. In our center, surgery is recommended in case of embolization contraindication or failure or in patients with recurrence of the fistula following embolization.
\end{abstract}

Keywords: Fistulas; Spinal cord; embolization; Spinal dural

\section{Introduction}

Spinal dural arteriovenous fistulas (DAVF) have first been identified in the $80^{\text {th }}[1,2]$. They are defined by the presence of an arteriovenous shunt seated in the spinal dura matter that connects a dural artery with a radicular vein. Edematous myelopathy is the main manifestation of spinal DAVFs and is caused by the increased pressure in the arterialized anterior and/or the posterior spinal vein(s) [3-6]. Spinal DAVFs are a rare and underdiagnosed pathology. Rapid diagnosis and treatment are, however, major determining factors for the outcome of the disease. The spontaneous course of this pathology can result in a substantial degree of morbidity that may be irreversible.

\section{Clinical Presentation}

Spinal DAVFs tend to affect middle aged men, which is suggestive of an acquired pathology. They manifest clinically as a progressive myelopathy $[1,3]$. The symptoms can rapidly become worse, and this can sometimes take place following a strenuous effort; a change in position; or occur spontaneously. Contrary to intracranial DAVFs, hemorrhage is not the way that spinal DAVFs come to light [7]. A delay in diagnosis occurs often, with an average diagnostic limbo of 10 to 15 months $[8,9]$. The neurological prognosis depends on the initial clinical condition and also on an early therapeutic treatment [10]. Spontaneous course of this pathology can result in irreversible para- or tetraplegia.

\section{Anatomy and Pathophysiology}

Vascularization of the spinal cord and its coverings is metameric in nature. At the medullar level, a distinct median anterior spinal axis (e.g. anterior spinal artery (ASA)) and two lateral posterior spinal axes (e.g. posterior spinal arteries (SPA)) run lengthwise along the spinal cord. In contrast to the radiculo-medullary arteries, the dural arteries are present on the right and left sides at each level. Some of these continue on as anterior or posterior radiculo-medullary arteries that vascularize the spinal cord through the ASA or PSA. There are anastomoses between radicular arteries in a longitudinal and cross-sectional manner (Figure 1). They vascularize the medullary coverings and the spinal nerves at each segment. These arteries arise, from the cervical to the sacral level, from the vertebral, external carotid (e.g. the ascending pharyngeal artery, occipital artery), cervical, costo-cervical, intercostal, lumbar, iliolumbar, and sacral arteries. The medullary venous drainage is variable and usually occurs along two longitudinal axes that are the anterior and posterior spinal veins. The radicular exit routes correspond to the radicular veins, which, like the radiculo-medullary arteries, are variable and not always present at each level. The intradural trajectory

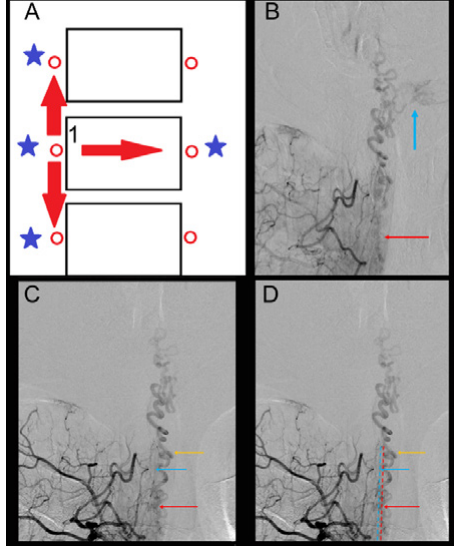

Figure 1: Thoracic DAVF with arterial feeding from the right T2-T3 intercostal artery in a 57 years old man presenting with a left C8 neuralgia without myelopathy.

A: Surgery is preferred to embolization in our institute if a radiculo-medullary artery arises from the lumbar or intercostal arteries at the level of the fistula (1) homo and contra laterally and homolaterally at the levels that lie above and below (Blue stars) because of the presence of dural anastomoses (Red arrows). $B$ : DAVF draining into the spinal veins with a radicular vein emerging from the C7-T1 foramen (Blue arrow). The venous ectasia is closed to the emergence of the left C8 nerve and explain the clinical presentation. The unrestricted venous drainage of the fistula may explain the absence of congestive myelopathy. Note the presence of an anterior radiculo-medullary artery at the level of the fistula (Red Arrow).

C and D: Anterior (Red Arrow) and posterior (Blue Arrow) radiculo-medullary arteries arise from the arterial feeding of the fistula. The patient was proposed to surgery because of the risk of embolic material migration in the anterior or the left posterior spinal arteries during embolization.

of radicular veins is on the order of $1 \mathrm{~cm}$. The dural spinal fistula is seated at this level [11].

Two types of fistula can have a peri-medullary venous drainage: spinal DAVF, for which the shunt point is seated in the spinal dura

*Corresponding author: Dr Stéphanie Lenck, Department of Neuroradiology, Hôpital Lariboisière, 2 rue Ambroise Paré, 75010 Paris, France, Tel: + 33 (0)1 499561 35: Fax: + 33 (0)1 499583 56; E-mail: stephanie.lenck@orange.fr

Received January 28, 2016; Accepted February 16, 2016; Published February 18,2016

Citation: Lenck S, Bernat A, Bresson D, Labeyrie M, Saint-Maurice J, et al. (2016) Spinal Dural Arteriovenous Fistula. J Spine 5: 287.doi:10.4172/2165-7939.1000287

Copyright: ( 2016 Lenck S, et al. This is an open-access article distributed under the terms of the Creative Commons Attribution License, which permits unrestricted use, distribution, and reproduction in any medium, provided the original author and source are credited. 
matter, and type 5 intracranial fistulas, for which the shunt point is intracranial [12]. The venous hyper pressure induced by arterialization of the anterior and/or posterior spinal veins hinders venous return to the spinal cord, thus resulting in a congestive myelopathy [4]. Thrombosis of a radicular vein can aggravate the myelopathy by restricting venous drainage of the fistula. Conversely, if there are enough functional radicular veins, the fistula may be asymptomatic. This physiopathology explains the lack of a correlation between the level of the fistula and the site where the medulla has become compromised.

\section{Imaging-based Diagnosis}

By providing a T2 sequence hyper signal of the spinal cord, medullary MRI usually allows a diagnosis of a dural fistula to be made [13-15]. The hyper signal most often extends over more than 2 vertebrae and involves the terminal cone for approximately $80 \%$ of cases [16]. As mentioned above, the level of medullar hyper signal does not provide an indication of the localization of the shunt. The presence of flow voids and vascular dilation in T2 sequence is highly suggestive of DAVF. In the absence of draining vein dilation, injection of gadolinium may be useful to achieve an enhanced contrast of the perimedullary spaces needed to make a diagnosis [15]. Medullary angio MRI can allow the level of the fistula to be localized and thus guide the medullar angiography [17].

An extended medullary hyper signal without vascular dilation can also reveal a DAVF when the venous drainage of the fistula is particularly restricted. These fistulas impact the most at the neurological level, since the pressure in the draining vein is generally very high. The presence of a spinal or intracranial DAVF is a counter indication for a lumbar puncture due to the risk of hemorrhaging stemming from the presence of dilated perimedullary vessels and the risk of altering the venous drainage of the DAVF associated with change in the pressure of the cerebrospinal fluid $[18,19]$. In light of this, medullar angiography is systematically considered in our institute in case of medullary hyper signal that extends over more than two vertebrae, even in the absence of vascular dilations.

Medullar angiography is the gold standard in the management of spinal DAVF (Figures 1 and 2). It allows a definitive diagnosis to be made, and to perform a pre-therapeutic assessment. Each artery that can feed a spinal artery should be catheterized. Spinal DAVFs are usually seated at the lower thoracic or upper lumbar level $[5,8]$. Angiography is hence initiated by catheterization of the intercostal and then the lumbar arteries. A venous return is probed for systematically following injection of the anterior spinal artery (ASA). A selective injection is hence performed on the intercostal or lumbar artery that feeds the Adamkiewicz artery. The non-visualization of the venous return of the spinal cord is highly suggestive of arteriovenous shunt, and the examination must be performed as thoroughly as possible. The examination is completed by selective injection of the medial and lateral sacral arteries, as well as the ascending and deep cervical arteries. Lastly, if the examination is negative, a full cerebral arteriography is performed to rule out a type 5 intracranial dural fistula.

Once the dural fistula has been identified, the intercostal or lumbar arteries are selectively injected contra laterally at the same level and homolaterally at the levels that lie above and below. An anterior or posterior radiculo-medullary artery is probed for on these axes and the axis pertaining to the fistula. We have previously observed the presence of arterial anastomoses between the radicular arteries in the longitudinal and cross-sectional planes. Opening of an anastomosis and migration of a liquid embolus in the anterior or posterior radiculo-medullary artery could lead to medullary ischemia. In our institute, if such an anatomical

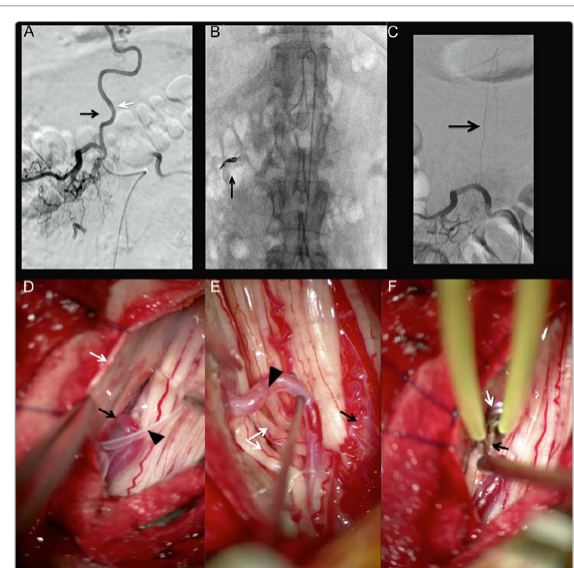

Figure 2: Lumbar DAVF with arterial feeding from the right L1 lumbar artery in a 57 years old man with progressive myelopathy.

A: Selective angiogram of the right L1 lumbar artery with arterialization of the draining vein of the DAVF (white arrow). Note the presence of a posterior radiculo-medullary artery arising from the same arterial feeding (black arrow).

$\mathrm{B}$ : Because of the presence of a posterior radiculo-medullary artery at the level of the DAVF, surgery has been preferred to embolization in this case. Thus, a coil has been implanted in the lumbar artery downstream of the DAVF to guide the surgical procedure (black arrow).

C: Post operative angiogram of the L1 lumbar artery. The fistula is cured and the posterior radiculo medullary artery has been respected during surgery (arrow)

D: Peri operative view of the fistula. Once the primary venous collector of the fistula has been identified (black arrow), the arachnoid mater (arrow head) and the roots are dissected as close as possible to the site where the dural vein emerges (black arrow). The dura mater is suspended (white arrow).

$\mathrm{E}$ : Per operative view of the fistula. We can see the cauda equina nerves roots (white arrows), the dilated perimedullary veins (black arrow) and the radicular vein corresponding to the primary venous collector of the fistula (black head arrow)

F: The draining vein has been occluded by a clip (white arrow) and by coagulation (black arrow)

feature is detected, surgery is preferable to embolization. In this regard, a coil is systematically implanted in the intercostal artery at the level of the fistula to guide the surgical procedure (Figure 2).

\section{Therapeutic Treatment}

\section{Endovascular treatment}

In our center, embolization of spinal dural fistulas is only performed if there is no anterior or posterior radiculo-medullary artery at the level of the fistula or homolaterally at the levels that lie above and below (Figure 2). This is systematically verified by taking angiographic pictures with apnea and under a general anesthetic. A selective 3 dimensional computed tomography with injection of the feeding artery is systematically performed in our institute. These technical settings allow the sensitivity of the examination to be increased compared to what can generally be achieved under local anesthetic, particularly in terms of detecting small caliber posterior radiculo-medullary arteries. The embolic agent that is used is a liquid embolus. The aim is for the embolic agent to spread from the afferent artery to at least $1 \mathrm{~cm}$ of the origin of the primary venous collector of the fistula (e.g. the radicular vein), while avoiding its migration into the spinal vein. If the primary venous collector is not occluded, it is highly likely that the dural fistula will recur by being reinjected through the anastomoses with the radicular arteries of the above and below or contralateral levels. Two types of liquid emboli can be used: Glue or Onyx. The cure rate with Glue varies from 44 to $100 \%$, depending on the series [20-23]. Dilution of the Glue is an important factor in determining the outcome for the treatment. It is diluted with Lipiodol ${ }^{\oplus}$, which gives it its radio-opacity. The more the Glue is concentrated, the faster it will polymerize; thus 
increasing the risk of it not reaching the primary venous collector and of proximally occluding the afferent artery. Conversely, too low a concentration can result in poor penetration of the Glue in the primary venous collector, and distal migration in the spinal vein. Based on our experience, the ideal dilution appears to be $1 / 3$ Glue with $2 / 3$ of Lipiodol $^{\circ}$ when the flow of the fistula is high, and $1 / 4$ Glue with $3 / 4$ of Lipiodol $^{\oplus}$ when the flow of the fistula is weaker. Ethylene vinyl alcohol copolymer $\left(\mathrm{Onyx}^{\oplus}\right)$ has been used recently to treat medullary DAVFs $[24,25]$. This embolic agent has the advantage of being more likely than Glue to reach the primary venous collector. In light of its capacity to open anastomoses, there must be a high level of certainty regarding the absence of radiculo-medullary arteries at the level of the fistula or at the homolateral levels that lie above and below. In our center it is used rarely since some radiculo-medullary arteries, and posterior ones in particular, are sometimes not visible by conventional angiography.

\section{Surgical treatment}

A surgical approach is recommended in case embolization is contraindicated, has failed or in patient with recurrence of DAVF after embolization $[26,27]$. The success rate of surgery is higher than that of embolization ( $98 \%$ vs $46 \%$ ) [28]. The patient is placed in a prone position and the level of the fistula is found by using a coil that is implanted in the corresponding intercostal artery upon performance of the angiographic assessment. The procedure is performed using a surgical microscope. The shunt is intradural, and most often seated on the posterior external side of the spinal dura mater at the level of the lower part of the vertebral pedicle. It can be seen extradurally as an arterial mass emanating from the radicular artery. After opening the middle of the dura mater, the intradural vein can be seen in respect to the shunt. It is most often dilated and arterialized. Intravenous injection of indocyanine green allows early injection and hence arterialization of the vein to be seen by fluorescence imaging with a surgical microscope. Once the primary venous collector of the fistula has been identified, the arachnoid mater and the roots are dissected as close as possible to the site where the dural vein emerges (Figure 2). The vein is occluded either by a clip or by coagulation. After the exclusion, the absence of arterialization of the vein is verified by a new test with indocyanine green.

The dura mater is then closed up again in a fully sealed manner and suspended in order to avoid an extradural hematoma.

\section{Post-embolization or post-operative treatment}

There is no consensus and there are no set procedures for the postembolization or post-operative treatment of spinal DAVFs. In our center, medullar angiography is routinely performed to verify healing of the DAVF in the 48 hours following surgery. If there is no improvement of the clinical disability within 6 weeks, or if a clinical deterioration occurs during the follow up, a medullar MR and a conventional DSA should be performed. A residual fistula, or more rarely a second fistula should be scrutinized [21].

In our experience, a curative anticoagulation is systematically prescribed for the four weeks following the intervention. A medullar MR is performed when curative anticoagulation is stopped to ensure the regression of venous dilatations. This measure is based on clinical experience and pathophysiological considerations. A significant number of patients exhibit a secondary worsening of symptoms after experiencing an initial improvement. The sudden reduction in flow in the dilated spinal veins can lead to thrombosis of the spinal veins or the radicular venous outlets. Angiography confirms the absence of a fistula recurrence, and whether the venous return of the ASA is either slowed or all together absent. Angiography can also reveal stagnation of one or more spinal veins with a lack or paucity of radicular exit routes linked with thrombosis of radicular veins. Medullary venous insufficiency can lead to irreversible venous congestive myelopathy. In case of recurrence of the symptoms, pan-medullary arteriography should be performed, preferably under general anesthesia, so as to rule out a recurrence of the initial DAVF or a new fistula. Venous insufficiency post-exclusion of a DAVF remains a diagnosis by elimination.

\section{Conclusions}

Spinal DAVFs are rare but also often underdiagnosed. Early diagnosis and treatment are, however, major prognostic factors. Proper interpretation of the MRI imaging, and particularly the medullar angiography, allow for an appropriate level of therapeutic intervention. In our center, surgery is recommended in case of embolization contraindication or failure or in patients with recurrence of the fistula following embolization. In case of recurrence of the symptoms, medullar angiography should be performed to rule out a recurrence of the DAVF, a new fistula, or a medullary venous insufficiency.

\section{References}

1. Symon L, Kuyama H, Kendall B (1984) Dural arteriovenous malformations of the spine. Clinical features and surgical results in 55 cases. J Neurosurg 60 238-247.

2. Mourier KL, Gelbert F, Rey A, Assouline E, George B, et al. (1989) Spinal dural arteriovenous malformations with perimedullary drainage. Indications and results of surgery in 30 cases. Acta Neurochir (Wien) 100: 136-141.

3. Jellema K, Tijssen CC, van Gijn J (2006) Spinal dural arteriovenous fistulas: a congestive myelopathy that initially mimics a peripheral nerve disorder. Brain 129: 3150-3164.

4. Jeng Y, Chen DY, Hsu HL, Huang YL, Chen CJ, et al. (2015) Spinal Dura Arteriovenous Fistula: Imaging Features and Its Mimics. Korean J Radiol 16 1119-1131.

5. Koch C (2006) Spinal dural arteriovenous fistula. Curr Opin Neurol 19: 69-75.

6. van Dijk JM, TerBrugge KG (2002) Dural arteriovenous fistulas. J Neurosurg 97: $1486-1487$

7. Koch C, Gottschalk S, Giese A (2004) Dural arteriovenous fistula of the lumba spine presenting with subarachnoid hemorrhage. Case report and review of the literature. J Neurosurg 100: 385-391.

8. Jellema K, Canta LR, Tijssen CC, van Rooij WJ, Koudstaal PJ, et al. (2003) Spinal dural arteriovenous fistulas: clinical features in 80 patients. J Neurol Neurosurg Psychiatry 74: 1438-1440.

9. van Dijk JM, TerBrugge KG, Willinsky RA, Wallace MC (2002) Multiplicity of dural arteriovenous fistulas. J Neurosurg 96: 76-78.

10. Cenzato M, Debernardi A, Stefini R, D'Aliberti G, Piparo M, et al. (2012) Spinal dural arteriovenous fistulas: outcome and prognostic factors. Neurosurg Focus 32: E11.

11. Lasjaunias PL, Berenstein A, Brugge KGt (2001) Surgical neuroangiography (2nd edn), Springer Berlin.

12. Cognard C, Gobin YP, Pierot L, Bailly AL, Houdart E, et al. (1995) Cerebra dural arteriovenous fistulas: clinical and angiographic correlation with a revised classification of venous drainage. Radiology 194: 671-680.

13. Gilbertson JR, Miller GM, Goldman MS, Marsh WR (1995) Spinal dura arteriovenous fistulas: MR and myelographic findings. AJNR Am J Neuroradio 16: 2049-2057.

14. Bowen BC, Fraser K, Kochan JP, Pattany PM, Green BA, et al. (1995) Spinal dural arteriovenous fistulas: evaluation with MR angiography. AJNR Am J Neuroradiol 16: 2029-2043.

15. Luetmer PH, Lane Jl, Gilbertson JR, Bernstein MA, Huston J, et al. (2005) Preangiographic evaluation of spinal dural arteriovenous fistulas with elliptic centric contrast-enhanced MR Angiography and effect on radiation dose and volume of iodinated contrast material. AJNR Am J Neuroradiol. 26: 711-718.

16. Hurst RW, Grossman RI (2000) Peripheral spinal cord hypointensity on 
T2-weighted MR images: a reliable imaging sign of venous hypertensive myelopathy. AJNR Am J Neuroradiol 21: 781-786.

17. Saraf-Lavi E, Bowen BC, Quencer RM, Sklar EM, Holz A, et al. (2002) Detection of spinal dural arteriovenous fistulae with MR imaging and contrast-enhanced MR angiography: sensitivity, specificity, and prediction of vertebral level. AJNR Am J Neuroradiol 23: 858-867.

18. Rastogi S, Liebeskind DS, Zager EL, Volpe NJ, Weigele JB, et al. (2004) Rapid cognitive decline following lumbar puncture in a patient with a dural arteriovenous fistula. Surg Neurol 62: 341-345.

19. Koerts G, Vanthuyne V, Delavallee M, Rooijakkers H, Raftopoulos C (2013) Spinal dural arteriovenous fistula presenting with paraplegia following lumbar puncture. J Neurosurg Spine 19: 57-60.

20. Van Dijk JM, TerBrugge KG, Willinsky RA, Farb RI, Wallace MC (2002) Multidisciplinary management of spinal dural arteriovenous fistulas: clinical presentation and long-term follow-up in 49 patients. Stroke 33: 1578-1583.

21. Hall WA, Oldfield EH, Doppman JL (1989) Recanalization of spinal arteriovenous malformations following embolization. J Neurosurg 70: 714-720.

22. Niimi Y, Berenstein A, Setton A, Neophytides A (1997) Embolization of spinal dural arteriovenous fistulae: results and follow-up. Neurosurgery 40: 675-682.

23. Westphal M, Koch C (1999) Management of spinal dural arteriovenous fistulae using an interdisciplinary neuroradiological/neurosurgical approach: experience with 47 cases. Neurosurgery 45: 451-457.

24. Carlson AP, Taylor CL, Yonas H (2007) Treatment of dural arteriovenous fistula using ethylene vinyl alcohol (onyx) arterial embolization as the primary modality: short-term results. J Neurosurg 107: 1120-1125

25. Adamczyk P, Amar AP, Mack WJ, Larsen DW (2012) Recurrence of "cured" dural arteriovenous fistulas after Onyx embolization. Neurosurg Focus 32: E12.

26. Huffmann BC, Gilsbach JM, Thron A (1995) Spinal dural arteriovenous fistulas: a plea for neurosurgical treatment. Acta Neurochir (Wien) 135: 44-51.

27. Afshar JK, Doppman JL, Oldfield EH (1995) Surgical interruption of intradural draining vein as curative treatment of spinal dural arteriovenous fistulas. J Neurosurg 82: 196-200.

28. Steinmetz MP, Chow MM, Krishnaney AA, Andrews-Hinders D, Benzel EC, et al. (2004) Outcome after the treatment of spinal dural arteriovenous fistulae: a contemporary single-institution series and meta-analysis. Neurosurgery 55 $77-87$ 